\section{Laterality effects in the method of triads*}

\author{
RALPH F. HALL \\ University of Sydney, Sydney, N.S.W. 2006, Australia
}

The method of triads for psychophysical judgments was analyzed in terms of the unidimensional unfolding model of Coombs (1964). This model predicts laterality effects on the stochastic transitivity of triples for which existing models have been unable to account. An experiment was conducted in which triadic judgments were made on circles differing in area. The results confirmed the stochastic transitivity predictions, but a quantitative formulation of the model proved unsatisfactory.

In the method of triads, $S$ is instructed to report which of two stimuli (the comparison stimuli) is more similar to a third stimulus (the standard stimulus). When the stimuli vary along a single physical dimension, the comparison stimuli can be classified according to whether they lie on the same side of the standard stimulus (unilateral pairs) or on opposite sides (bilateral pairs). Krantz (1967) argued that bilateral pairs present a more difficult discrimination task to the $\mathbf{S}$ than do unilateral pairs. This will be referred to as the laterality effect.

A theoretical analysis of the laterality effect was presented by Coombs (1958) in terms of his unfolding model of preferential choices. In this model the individual and stimuli are represented by points on a unidimensional preference scale. The point corresponding to the individual is called his "ideal point" and represents his point of maximum preference, It is assumed that when $\mathrm{S}$ is asked to choose between two stimuli he will choose the one that lies closer to his ideal point. By adding the assumption of randomly varying stimulus and ideal points, Coombs predicted that inconsistency of preference would be greater on bilateral pairs than on unilateral pairs. Although this model was not designed to account for similarity judgments using the method of triads, it can easily be modified to do so. All that is necessary is to substitute for the probability distribution of the individual's ideal point a probability distribution of the standard stimulus. The theoretical predictions remain unchanged by this translation of the model.

Coombs (1958) derived additional predictions from this model concerning stochastic transitivity of the choice proportions. He identified three kinds of triples of stimuli: unilateral triples, in which all three stimuli are on the same side of the

*This research was supported by a University of Sydney research grant. The author is indebted to Mrs. Carolyn Lowry for her assistance in the conduct of the study. standard; bilateral adjacent triples, in which two stimuli are from one side of the standard and the third is from the opposite side and is either closest to the standard or furthest away; and bilateral split triples, in which the stimulus from one side of the standard lies between the two stimuli from the other side in distance from the standard. Since bilateral pairs are more difficult to discriminate than unilateral pairs, it is to be expected that bilateral adjacent triples will produce violations of strong stochastic transitivity (SST). That is, if $i, j$, and $k$ are stimuli and $p_{i j}$, $p_{j k}$, and $p_{i k}$ are the choice proportions $\left(p_{i j}\right.$ referring to the proportion of times $i$ is chosen as more similar to the standard than $\mathrm{j}$ ), then in a bilateral adjacent triple, $\mathrm{i}$ and $\mathrm{k}$ always form a bilateral pair, whereas one of the other pairs (say, $i$ and $j$ ) will be a unilateral pair. The kind of violation of SST predicted is that the proportion, $p_{i k}$. for the bounding bilateral pair will be less than the proportion, $p_{i j}$, for the unilateral pair. Hence, bilateral adjacent triples should satisfy at least moderate stochastic transitivity (MST). Bilateral split triples and unilateral triples should satisfy SST since the bounding pair is unilateral in both cases, the other two being bilateral in the case of a bilateral triple and unilateral in the case of a unilateral triple. To test these predictions, Coombs (1958) instructed Ss to rank shades of gray according to how closely they corresponded to orders were converted into pairwise proportions, and the above predictions concerning stochastic transitivity confirmed. The experiment was repeated (Coombs, 1964), using paired comparisons, and the same results obtained. The only respect in which this experiment differs from the method of triads is that the $S$ is asked to define his own standard (i.e., an "ideal" shade of gray) rather than being provided with a standard.

Unlike most psychophysical scaling models (e.g., Thurstone, 1927; Luce, unfolding theory is stated in qualitative form only. Bechtel (1968) their ideal shade of gray. The rank 1959 , 1961), this application of has developed a quantitative version of the model, in which the stimuli are fixed points along the preference scale and only the ideal point varies randomly. This makes the model an unlikely contender as a model for the method of triads, since it assumes a probability distribution for the standard stimulus but not for the comparison stimuli. However, it does predict the laterality effects found by Coombs.

The present experiment was conducted to test both the qualitative predictions concerning laterality effects derived by Coombs (1958, 1964) and the quantitative predictions derived from Bechtel's model. As Krantz (1967) found, models that make no allowance for the laterality effect are unsatisfactory as models for the method of triads.

\section{Subjects}

\section{METHOD}

Sixty students from the introductory psychology course at the University of Sydney served as Ss. Participation in experiments was part of the course requirement.

\section{Apparatus}

The $\mathrm{S}$ was seated at a table, facing a screen situated on the wall of the laboratory at a distance of $2.29 \mathrm{~m}$ from the $S$. The stimuli, circles differing in area, were projected onto the screen by a Carousel slide projector. Each slide contained three circles in triangular formation, with the standard stimulus at the top and the two comparison stimuli situated symmetrically beneath the standard. The projection of the standard on the screen measured $5.2 \mathrm{~cm}$ in radius, and eight comparison stimuli were used with radii $3.1,3.7,4.2,4.8,5.5,5.7$, 6.8 , and $7.5 \mathrm{~cm}$. Thus, four comparison stimuli were smaller in area than the standard and four were larger. The projector was situated on a table to the side of the seated $S$, and a three-position spring-loaded switch was mounted on the table in front of the S. A photoelectric cell in the projector closed a relay, which activated a chronoscope as soon as the slide dropped into place. The chronoscope was stopped by the S's deflecting the switch in either direction, thus enabling measurement of S's reaction time. A decade interval timer controlled the time interval between S's response and the next slide. A curtain separated $E$ from $S$, and red and green lights were situated in front of $\mathbf{E}$ to enable identification of the response made by $S$.

\section{Procedure}

All pairs of the eight comparison stimuli were presented, including the 
Table 1

Ordered Proportions of Choices of Row Over Column Stimuli

Stimulus on Right

\begin{tabular}{|c|c|c|c|c|c|c|c|c|c|}
\hline & \multicolumn{8}{|c|}{ Stimulus on Right } \\
\hline & & A & $\mathbf{B}$ & C & D & $\mathbf{E}$ & $\mathbf{F}$ & $\mathbf{G}$ & $\mathbf{H}$ \\
\hline \multirow{8}{*}{ 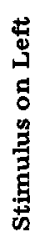 } & $\mathbf{A}$ & - & .000 & .000 & .000 & .000 & .017 & .033 & .150 \\
\hline & $\mathbf{B}$ & .983 & - & .033 & .017 & .000 & .017 & .167 & .550 \\
\hline & $\mathbf{C}$ & 1.000 & .833 & - & .017 & .000 & .017 & .417 & .850 \\
\hline & $\mathbf{D}$ & 1.000 & .983 & .983 & - & .033 & .183 & .883 & .583 \\
\hline & $\mathbf{E}$ & .983 & 1.000 & .933 & .867 & - & .733 & .917 & 1.000 \\
\hline & $\mathbf{F}$ & .983 & 1.000 & .917 & .367 & .300 & - & .933 & 1.000 \\
\hline & $\mathbf{G}$ & .867 & .867 & .417 & .100 & .000 & .067 & - & .983 \\
\hline & $\mathbf{H}$ & .817 & .517 & .183 & .017 & .017 & .017 & .017 & - \\
\hline
\end{tabular}

Table 2

Predicted and Obtained Degrees of Stochastic Transitivity Satisfied by the Three Kinds of Triples

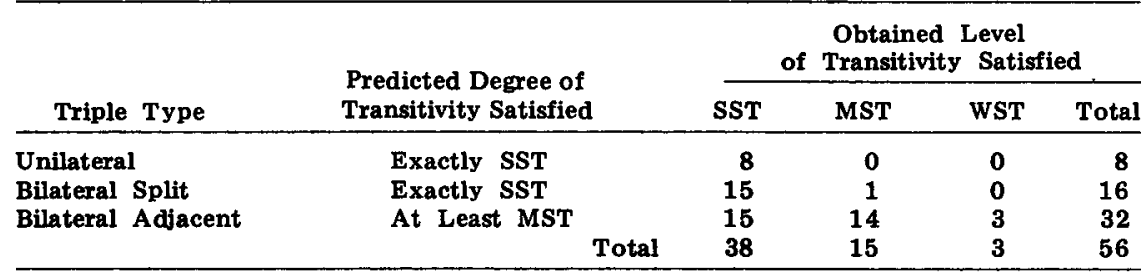

Table 3

Folded and Unfolded Scale Values for the Eight Comparison Stimuli

\begin{tabular}{ccccccccc}
\hline Stimulus & A & B & C & D & E & F & G & H \\
\hline $\begin{array}{c}\text { Unfolded Scale } \\
\quad \text { Value }\end{array}$ & -3.905 & $-\mathbf{3 . 0 6 2}$ & -2.062 & -1.196 & -0.418 & .026 & 2.265 & 3.384 \\
$\begin{array}{c}\text { Folded Scale } \\
\text { Value }\end{array}$ & $\mathbf{3 . 9 0 5}$ & $\mathbf{3 . 0 6 2}$ & $\mathbf{2 . 0 6 2}$ & $\mathbf{1 . 1 9 6}$ & .418 & .026 & 2.265 & 3.384 \\
\hline
\end{tabular}

Table 4

Mean Reaction Times for the Ordered Triadic Judgments

Stimulus on Right

\begin{tabular}{|c|c|c|c|c|c|c|c|c|c|}
\hline & & & & & timu & on Rig & & & \\
\hline & & $\mathbf{A}$ & $\mathbf{B}$ & C & $\mathbf{D}$ & $\mathbf{E}$ & $\mathbf{F}$ & $\mathbf{G}$ & $\mathbf{H}$ \\
\hline & $\mathbf{A}$ & - & 1.386 & 0.991 & 1.145 & 1.085 & 1.228 & 1.830 & 1.910 \\
\hline 3 & $\mathbf{B}$ & 1.534 & - & 1.353 & $\mathbf{1 . 1 3 5}$ & 1.116 & 1.232 & 1.967 & 2.223 \\
\hline 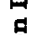 & C & 1.375 & 2.027 & - & 1.357 & 1.059 & 1.431 & 1.790 & 1.850 \\
\hline 0 & $\mathbf{D}$ & 1.095 & 1.329 & 1.374 & - & 1.271 & 1.501 & 1.642 & 1.779 \\
\hline 3 & $\mathbf{E}$ & 1.070 & 1.135 & 1.165 & 1.359 & - & $\mathbf{1 . 8 9 4}$ & 1.392 & 1.201 \\
\hline 3 & $\mathbf{F}$ & 1.192 & 1.398 & 1.472 & 1.974 & 2.086 & - & 1.435 & 1.149 \\
\hline 8 & $\mathbf{G}$ & 1.725 & 2.152 & 1.872 & 1.579 & 1.195 & 1.452 & - & 1.652 \\
\hline$\ddot{D}$ & $\mathbf{H}$ & 2.181 & 2.611 & 1.900 & 1.321 & 1.182 & 1.157 & 1.564 & - \\
\hline
\end{tabular}

two orders for each pair, making 56 presentations. The $\mathrm{S}$ was told that each slide would contain three circles in triangular formation and that he was to judge which of the two lower circles was more similar in area to the one above. $S$ made his judgment by deflecting the switch to the left if he judged the left-hand circle to be more similar in area to the standard, and to the right if he chose the right-hand circle. This enabled measurement of reaction times as well as of the actual choice made. The 56 slides were presented in a random order, the same random order being used for all Ss. The time interval between the S's response and the next slide was fixed at 5 sec to allow $E$ time to record the response made and the reaction time.

\section{RESULTS}

The matrix of choice proportions is the standard was derived. This order was EFDCGBHA, which differs from the actual physical order of the circles in terms of differences in area (or radius) from the standard. This latter order is EDFCBGAH. There is a bias toward judging circles larger than the standard as less larger than smaller circles are smaller. This is consistent with a logarithmic function (or any negatively accelerated function) relating subjective area to physical area (i.e., Fechner's law).

The types of triples and the degree of stochastic transitivity satisfied are shown in Table 2. With eight stimuli, there are

$$
\left(\begin{array}{l}
8 \\
3
\end{array}\right)=56 \text { triples, }
$$

of which 8 are unilateral, 16 are bilateral split, and 32 are bilateral adjacent triples. In only 4 of the 56 triples was the predicted degree of stochastic transitivity not satisfied. Of the 14 violations of SST that satisfied MST for bilateral adjacent triples, 13 were of the type predicted. That is, the pairwise proportion for the bounding bilateral pair was less than that for the embedded unilateral pair.

To test Bechtel's quantitative version of the unfolding model, least-squares estimates of the scale values of the comparison stimuli were calculated by the method described by Bechtel (1968) for the response biased model. The scale values for both the folded and unfolded scales are shown in Table 3. The unfolded scale preserved the order of magnitude of the comparison stimuli, but the folded scale did not produce the same order as was derived from the pairwise proportions. The order on the folded scale was FEDCGBHA, which exhibits a number of differences from the rank order given above. The zero point, which corresponds to the standard, actually lies between $D$ and $E$. Thus, Bechtel's model does not even preserve the rank order of the judged similarity of the comparison stimuli to the standard.

The mean reaction times (RTs) for each pair are shown in Table 4. The 4 by 4 top- and bottom-left submatrices represent mean RTs for unilateral pairs. The mean $\mathrm{RT}$ for all bilateral pairs (1.594) exceeded that for unilateral pairs (1.394), confirming the greater difficulty of discrimination for bilateral pairs. That RTs reflect difficulty of discrimination can be observed in Fig. 1, in which mean RT is plotted against the absolute value of the deviation of the pairwise proportions from .5. In this graph, small deviations represent highly confusable pairs, whereas the maximum deviation of .5 represents 


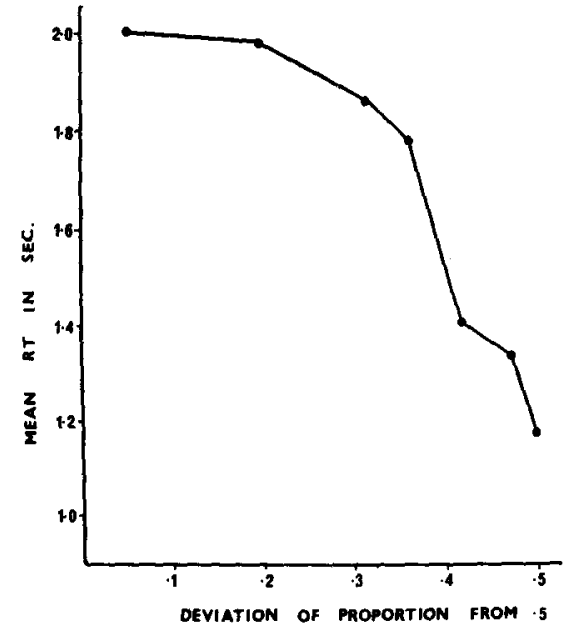

Fig. 1. Mean RT as a function of difficulty of discrimination measures by $\left|p_{i j}-.5\right|$.

perfectly discriminable pairs. The relationship obtained shows that $R T$ decreases as difficulty of discrimination decreases.

\section{DISCUSSION}

The results of this experiment provide considerable support for this application of the unfolding model to the method of triads. The predictions concerning stochastic transitivity of triples were confirmed, with only a few exceptions. However, the quantitative version of the model developed by Bechtel (1968) proved unsatisfactory due to a lack of correspondence between the rank orders derived from the folded scale and the pairwise proportions. This discrepancy is also present in the second set of illustrative data presented by Bechtel, although he makes no comment on it. The most likely reason for the failure of this model is the unreasonable assumption that the comparison stimuli are fixed points on the judgmental scale, whereas the standard stimulus projects a probability distribution (discriminal process, to use Thurstone's terminology) on the scale. This problem could be overcome by developing a model in which probability distributions are associated with both the comparison stimuli and the standard. An outline of such a model has already been sketched by Luce and Galanter (1963, p. 252). The results of the present study suggest that further developments in this direction may provide a satisfactory psychophysical model for triadic judgments. The finding that RT is systematically related to difficulty of discrimination also suggests that such a model could be generalized to incorporate a theoretical account of RTs.

\section{REFERENCES}

BECHTEL, G. Folded and unfolded scales from preferential paired comparisons. Journal of Mathematical Psychology, $1968,5,333-357$.

COOMBS, C. H. On the use of inconsistency of preferences in psychological measurement. Journal of Experimental Psychology, 1958, 55, 1-7.

COOMBS, C. H. A theory of data. New York: Wiley, 1964.

KRANTZ, D. $\cdot$. Rational distance functions for multidimensional scaling. Journal of Mathematical Psychology, 1967, 4, 226-245.

LUCE, R. D. Individual choice behavior New York: Wiley, 1959.

LUCE, R. D. A choice theory analysis of similarity judgments. Psychometrika, $1961,26,151-163$.

LUCE R D. \& GALANTER, E. Psychophysical scaling. In R. D. Luce et al (Eds.), Handbook of mathematical psychology. Vol. I. New York: Wiley, 1963.

THURSTONE, L. L, Psychophysical analysis. American Journal of Psychology, 1927, 38, 368-389.

(Accepted for publication December 7, 1970.) 